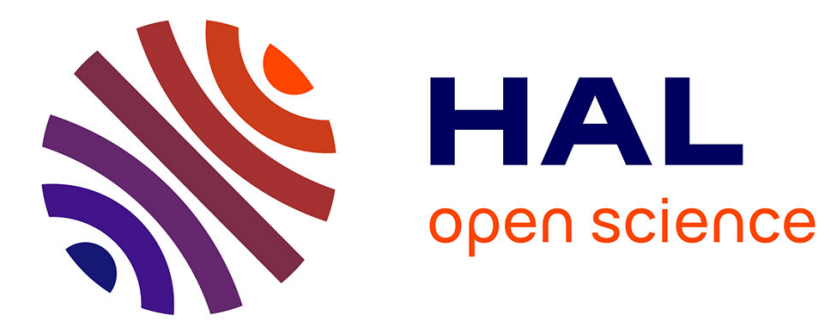

\title{
Buddha et la marche sur les eaux : du prodige à la condition de l'arhant
}

Guillaume Ducoeur

\section{To cite this version:}

Guillaume Ducoeur. Buddha et la marche sur les eaux: du prodige à la condition de l'arhant. Archimède : archéologie et histoire ancienne, 2019, 6, pp.29-38. halshs-02894232

\section{HAL Id: halshs-02894232 \\ https://shs.hal.science/halshs-02894232}

Submitted on 8 Jul 2020

HAL is a multi-disciplinary open access archive for the deposit and dissemination of scientific research documents, whether they are published or not. The documents may come from teaching and research institutions in France or abroad, or from public or private research centers.
L'archive ouverte pluridisciplinaire HAL, est destinée au dépôt et à la diffusion de documents scientifiques de niveau recherche, publiés ou non, émanant des établissements d'enseignement et de recherche français ou étrangers, des laboratoires publics ou privés. 


\section{ARCHIMĖDE $N^{\circ} \mathbf{6}$

DOSSIER THÉMATIQUE :

HISTOIRES DE FIGURES CONSTRUITES : LES FONDATEURS DE RELIGION

1 Guillaume DUCCEUR

Introduction

9 Philippe SWENNEN

Zarathushtra, une construction liturgique

16 Kyong-Kon KIM

Laozi. De la figure du maître mythique à la divinité taoïque

29 Guillaume DUCCEUR

Buddha et la marche sur les eaux : du prodige à la condition de l'arhant

39 Jean-Marie HUSSER

Le martyre de Jean-Baptiste, préfiguration de la Passion du Christ ?

48 Michel TARDIEU

Héraclès/Jésus dans la sophistique impériale

55 Anne-Sylvie BOISLIVEAU

Mahomet entre exégèse, hagiographie, rite et histoire

71 DOSSIER THÉMATIQUE :

JOUER DANS L'ANTIQUITÉ : IDENTITÉ ET MULTICULTURALITÉ GAMES AND PLAY IN ANTIQUITY: IDENTITY AND MULTICULTURALITY

213 VARIA 


\section{BUDDHA ET LA MARCHE SUR LES EAUX : DU PRODIGE À LA CONDITION DE L'ARHANT}

\section{Guillaume DUCOEUR}

Professeur en histoire des religions

Université de Strasbourg UMR 7044 Archimède gducoeur@unistra.fr

\section{RÉSUMÉ}

Les Vinaya des écoles du Sthaviravāda conservent des épisodes de la vie du fondateur du bouddhisme qui participent de sa figure construite non-historique visant à mettre en scène et à rendre vivantes les règles disciplinaires imposées aux membres de la communauté des bhikșu. L'épisode de la

MOTS-CLÉS

Buddha, marche, eau,

biographie,

miracle,

arhant,

Nairañjanā,

Sāñcī,

Kāśyapa,

bouddhisme. conversion de Kāśyapa d'Uruvilvā dans lequel le Buddha marche sur les eaux de la Nairañjanā illustre symboliquement les capacités surnaturelles acquises après l'éveil tout en rappelant que le but ultime de tout bhikșu demeure l'obtention de la condition de l'arhant.
The Vinaya of the Sthaviravāda schools contain stories about the life of the founder of Buddhism that pertain to his nonhistorical constructed figure aimed at portraying and living the disciplinary rules imposed on the members of the bhikșu's community. The storie of the conversion of Kāśyapa of Uruvilvā in which the Buddha walks on the waters of the Nairañjanā symbolically illustrates the supernatural abilities acquired after the awakening while reminding that the final aim of all bhikșu remains the obtaining of the condition of the Arhant.
KeYWORDS

Buddha, walk, water, biography, miracle, arhant, Nairañjanā, Sāñcī, Kāśyapa, Buddhism. 
Depuis le ve s. av. J.-C., la communauté bouddhique (samgha) a produit un grand nombre de récits traditionnels sur la vie de son fondateur qui firent l'objet de représentations figurées au moins dès le $\mathrm{II}^{\mathrm{e}} \mathrm{s}$. av. J.-C. Aux Sūtra et Vinaya du canon des écoles anciennes (sthaviravāda), qui relatent pour certains quelques fragments de sa vie déjà construite de longue date, se sont adjoints des récits de vies antérieures (Jātaka) et d'exploits glorieux (Avadāna) ainsi que des biographies autonomes partielles ou complètes (Carita). Le plein essor de ces biographies autonomes et de l'art figuratif eut lieu entre le $\mathrm{I}^{\mathrm{er}} \mathrm{s}$. et le $\mathrm{III}^{\mathrm{e}} \mathrm{S}$. apr. J.-C. sous le règne des rois Kușāna en Inde du Nord et Śātavāhana en Inde centrale. Durant cette même période, les tenants du mahāyāna procédèrent à la relecture de la vie traditionnelle du fondateur en fonction des différents éléments novateurs de leur doctrine.

Ces éléments narratifs abondants, pour la plupart emplis de merveilleux, n'ont guère de valeur historique et certains religieux-mendiants (bhikșu) bouddhistes [1] en vinrent eux-mêmes à s'interroger sur I'historicité de leur propre fondateur, l'Éveillé (buddha) dont on répétait génération après génération qu'il avait atteint l'extinction complète (parinirvāṇa) au moment de sa mort. De fait, le Buddha historique échappe à celui qui tente d'en restituer la vie, car les sources textuelles bouddhiques ne sont nullement des récits d'événements historiques, leurs auteurs n'ayant jamais prétendu faire de I'histoire au sens où l'entend la discipline historique moderne. Ceci induit que les épisodes de la vie du Buddha ont avant tout une valeur symbolique et surtout didactique fondée sur la doctrine bouddhique qu'ils exposent et rendent vivante avec force conviction auprès des croyants (śraddhin). Le Buddha qui y est décrit incarne donc, pour le véhicule des auditeurs (śrāvakayāna) des écoles anciennes, le bhikșu idéal typique montrant la voie à suivre afin d'atteindre l'extinction (nirvāṇa) de la soif (tṛṣnāa d'existence ou, pour le véhicule des bodhisattva (bodhisattvayāna) prôné par les mahāyānistes, le bodhisattva archétypal indiquant le chemin vers le parfait et complet éveil (samyaksamboddhi).
Pourtant, dès les débuts du XIX ${ }^{e}$ s., les indianistes européens essayèrent d'extirper des épisodes biographiques les éléments merveilleux, s'imaginant restituer ainsi une possible trame narrative historique. Le bouddhologue allemand Hermann Oldenberg (18541920), alors professeur à I'Université de Kiel, était convaincu d'y parvenir en ne gardant des sources textuelles pālies que les seuls éléments narratifs les plus rationnels et affirmait alors en réaction à l'approche mythologique [2] de I'indianiste Émile Senart (1847-1928) qui travaillait, quant à lui, sur les textes bouddhiques en langue sanskrite :
« La critique historique ne peut pas, là où il n'y a que des probabilités, créer des certitudes. Que chacun décide ou s'abstienne de décider, comme il le trouvera bon ; pour ma part, qu'il nous soit permis de professer l'opinion que nous possédons dans le récit de la manière dont le descendant des Sakyas est devenu le Bouddha, un véritable fragment d'histoire. » [3]

En 1949, Alfred Foucher (1865-1952), qui étudia, durant sa carrière académique, la vie du fondateur du bouddhisme à travers les productions artistiques indiennes et centrasiatiques, avait bien saisi l'impossibilité de telles restitutions et déclara au sujet de ces approches mythologique et rationaliste :

\begin{abstract}
«Dans le Bouddha d’E. Senart c'est I'homme qui manque ; dans celui d'Oldenberg ce qui fait défaut, c'est le dieu. Or, ne nous lassons pas de le répéter, bien que Çâkya-mouni ait lui-même pris soin de nous avertir qu'il n'était qu'un homme, il est non moins certain que l'Inde en a fait un dieu. C'est là le trait dominant de sa destinée, là que réside I'intérêt passionnant de son histoire ; et quiconque en entreprend une étude d'ensemble ne saurait négliger ni I'un ni l'autre de ces deux aspects. » [4]
\end{abstract}

Et, si les brāhmanes se présentaient comme des hommes-dieux (manușyadeva [5]) dans la société indo-ārya, si le mari était la divinité de l'épouse (patir hi devatā nāryās [6]), le Buddha était, quant
[1] P[ali] T[ext] S[ociety], Milindapañha 70 et 73.

[2] SENART 1875.

[3] Oldenberg 1903, p. 115.
[4] FOUCHER 1949, p. 13.

[5] Śatapatha Brāhmaña 2.2.2.6.

[6] Rāmāyaña 7.48.17c. 
à lui, la divinité de ses disciples réunis en communauté, à l'égal de nombre de maîtres du courant des ascètes (śramana) qui proposaient à tout un chacun de suivre et d'expérimenter leur voie qui mènerait les êtres humains à la délivrance (mokșa) de la condition saṃsārique. Aussi les membres du saṃgha construisirent-ils la figure de leur maître (śāstṛ) au fur et à mesure des siècles et en fonction des nécessités que leur imposèrent des dissensions internes (samghabheda), des innovations doctrinales, des implantations territoriales nouvelles et toujours de plus en plus éloignées du Magadha, des demandes de protections royales, des rivalités avec d'autres courants religieux non-bouddhiques, des invasions étrangères, etc. Autant de facteurs historiques qui concoururent à enrichir la figure construite du Buddha.

Parmi les nombreux épisodes de la vie du fondateur, nous avons choisi de revenir sur celui de la marche sur les eaux qui intéresse particulièrement I'histoire comparée des religions. Depuis la fin du $\mathrm{XIX}^{\mathrm{e}}$ siècle, en effet, dans le double contexte de la découverte des textes bouddhiques et des prémices de la crise moderniste, cet épisode bouddhique a moins été étudié pour lui-même que par rapport à la péricope de Jésus marchant sur les eaux [7] (Mc 6.45-52; Mt 14.22-33; Jn 6.16-21). Notre présente contribution, quant à elle, aura pour objectif premier de restituer, tant faire se peut, I'histoire rédactionnelle d'un tel exploit glorieux (avadāna), comme nous l'avons déjà fait pour d'autres passages biographiques [8], et de mieux saisir les éléments doctrinaux et surtout disciplinaires qui sont au fondement de sa construction. Forts de cette recherche préalable à toute approche comparative, nous poursuivrons l'étude de cet épisode bouddhique en le confrontant avec la péricope néotestamentaire dans une future contribution.

\section{LES SOURCES TEXTUELLES ET ICONOGRAPHIQUES}

L'épisode du Buddha marchant sur les eaux d'une vaste inondation est conservé dans les Vinaya de différentes écoles anciennes, à savoir les Theravādin, les Mahišãsaka et les Dharmaguptaka, ainsi que dans l'Ekottarāgama issu probablement soit d'une des écoles de la branche des Mahāsāṃghika soit de celle des Sarvāstivādin. Il existe également d'autres récits dans lesquels soit un être créé par le Buddha marche sur les eaux d'une rivière soit un dévot (upāsaka) bouddhiste traverse une rivière en marchant dessus. Toutes ces versions proviennent des écoles anciennes et particulièrement de la branche des Vibhajyavādin ou Distinctionnistes. Le seul texte en langue indienne se trouve dans le Groupe des grands [textes] (Mahāvagga) de la Section (Khandhaka) des Vinaya [9] des Theravādin dont le canon scripturaire aurait été mis, selon la tradition bouddhique, par écrit sur feuilles de palmier au $\mathrm{I}^{\mathrm{er}} \mathrm{s}$. av. J.-C. sous le règne de Vatțagāmani (mort en 77 av. J.-C. ), roi d'Anuradhapura au Śrī Lańkā. Appartenant au chapitre premier, l'épisode suit le récit de l'éveil du Buddha et des premières conversions à sa doctrine.

Les trois autres sources sont des traductions chinoises de Vinaya en langues indiennes aujourd'hui perdues. Issus de la même branche des Vibhajyavādin, les Mahīśāsaka se distinguèrent des Theravādin, au cours des $\mathrm{III}^{\mathrm{e}}$-II ${ }^{\mathrm{e}} \mathrm{S}$. av. J.-C., et $\mathrm{s}^{\prime}$ implantèrent en Inde centrale avant d'essaimer dans le Nord-Ouest indien et au Śrī Lańkā. Leur Vinaya est connu par I'intermédiaire d'une traduction chinoise [10] réalisée par le moine kaśmīrien mahiśāsaka Buddhajīva (Fotuoshi, 佛陀什) et par Daosheng (道生) à Jiankang (actuelle ville de Nankin), en 423 apr. J.-C., sous la dynastie Song du Sud. Le Vinaya de l'école des Dharmaguptaka, issue de celle des Mahīśāsaka au cours des $\mathrm{II}^{\mathrm{e}-\mathrm{I}^{\mathrm{er}}}$ S. av. J.-C., a, quant à lui, été traduit en chinois [11] par Fonian (佛念) d'après la récitation qu'en fit le moine kaśmīrien Buddhayaśas (Fotuoyeshe, 佛陀耶舍) vers 410 apr. J.-C. à Chang'an (長安， actuelle ville de Xi'an). Enfin, une version est également conservée dans l'Ekottarāgama en traduction chinoise [12] qui fut réalisée par le moine centrasiatique Dharmanandin (量摩難提, Tanmonanti) en 384 apr. J.-C. puis révisée par Saṃghadeva (僧伽 提婆, Sengqietipo) en 397 ap. J.-C. Ce texte a une histoire rédactionnelle assez complexe, en raison notamment des additions mahāyāniques, et a été classé par certains bouddhologues parmi les œuvres des Mahāsāṃghika [13]. Quoi qu'il en soit, cet Āgama reflète une version pré-mahāyānique ayant appartenu à l'une des écoles bouddhiques anciennes.

[7] Lillie 1893 ; Carus 1897; Garbe 1914 ; SAINTYVES 1922 ; BROWN 1928, etc.

[8] DUCGUR $2011 ; 2014$ et 2019.

[9] PTS, Vinaya-pițaka, vol.1, Mahāvagga 1.20.16, p. 32.

[10] Vinaya en cinq sections ([Pañcavargikavinaya], Wufen Iu, 五分律), T[aishō Shinshū Daizōkyō]. 1421, vol. 22, p. $108 a 3$ et suivantes.

[11] Vinaya en quatre sections ([Caturvargikavinaya], Sifen lu, 四分律)，T. 1428, vol. 1, p. 567a4 et suivantes.

[12] Textes traditionnels des un et plus ([Ekottarāgama]，增壹阿含經，Zengyi ahan jing)，T. 125， vol. 2, p. $549 a 3$ et suivantes.

[13] PALumbo 2013. Sur les Mahāsāṃghika, voir en dernier lieu TOURNIER 2017. 
En ce qui concerne les biographies du Buddha partielles ou complètes, il est notoire que peu d'entre elles citent cet épisode. Le poète bouddhiste Aśvaghoșa n'en fit nulle mention dans son Buddhacarita qu'il composa dans la première moitié du $\mathrm{II}^{\mathrm{e}} \mathrm{s}$. apr. J.-C. [14] Il est néanmoins présent dans le Sūtra de la collection des actes passés du Buddha (Fo benxing ji jing, 佛本行 集經), traduction chinoise d'une biographie de l'école Dharmaguptaka réalisée par Jñānagupta (Shenajueduo, 闍那崛多) en 588 apr. J.-C.

Enfin, les sources bouddhiques présentent également des disciples du Buddha marchant sur l'eau. L'auteur de la partie introductive ou « exposé des occasions [des bonnes actions répétées] » (nidanakathā) du Silānisamsajātaka [15], rédigée au ve s. apr. J.-C., a repris ce thème de la marche sur l'eau, tout comme le rédacteur des exploits du Buddha qui glosa le Dharmapāda dans sa version chinoise (法句譬喻經, Faju piyu jing [16]) réalisée par Faju (法炬) entre 290 et 306 apr. J.-C.

Dans le domaine artistique, la représentation du Buddha qui réchappa à une soudaine montée des eaux est très rare [17]. Son absence dans le si riche répertoire figuratif gandhārien serait due, selon I'historien de l'art Alfred Foucher (1865-1952), à une difficulté technique, «jamais, en effet, les artistes indo-grecs n'ont tenté de représenter un fleuve en sculpture. Ils ont donc dû renoncer à figurer aux yeux, entre autres prodiges, celui que provoque le dévergondage intempestif de la Naïrañjanā, et dès lors il devient des plus vraisemblables que c'est à l'absence d'images de ce miracle qu'est dû l'oubli où il est tombé d'abord dans la tradition orale, puis dans la tradition écrite du Nord-Ouest » [18]. Ainsi s'explique probablement le silence du Mahāvastu de l'école des Mahāsāṃghika-Lokottaravādin et du Buddhacarita d'Aśvaghoșa sur cet épisode. Au contraire, en Inde centrale, parmi les nombreux exploits surnaturels ou miracles accomplis par le Buddha pour convertir Kāśyapa d'Uruvilvā et ses disciples, l'épisode de la montée des eaux a été choisi délibérément par le commanditaire du jambage de gauche de la porte (torana) est du grand stūpa de Sāñcī au I Ir s. av. J.-C. (fig. 1). Y ont ainsi été figurés par les sculpteurs indiens la montée des eaux submer- geant le tronc des grands arbres, les frères Kāśyapa embarqués à la recherche du Buddha et un espace symbolisant la zone sur laquelle le Buddha se promena sain et sauf. En 1918, I'archéologue John Marshall (1876-1958) en faisait la description suivante :

« La rivière Nairañjanā est montrée en crue
et Kāśyapa, accompagné de son disciple et du
batelier, se hâtant dans un bateau au secours
du Buddha. Puis, dans la partie inférieure de
l'image, Buddha, représenté par sa promenade
(chañkrama), apparaît marchant sur la surface
des eaux, et au premier-plan les figures de
Kāśyapa et son disciple sont une deuxième
fois reproduites, maintenant sur le sol sec et
rendant hommage au Maître (représenté par
le trône sur la droite, au coin inférieur) » [19].

Il ressort de cette brève présentation des sources que l'épisode lié à la conversion de Kāśyapa d'Uruvilvā remonte bien antérieurement au I ${ }^{\text {er }} \mathrm{s}$. av. J.-C., et qu'il a été conservé pour l'essentiel dans les Vinaya des écoles du sthaviravāda, et plus particulièrement parmi la branche des Vibhajyavādin, en Inde centrale. L'influence de sa représentation figurée, notamment celle conservée sur la porte est du stūpa de Sāñcī, est particulièrement éclairante sur la tradition rapportée par les écoles des Theravādin et des Dharmaguptaka, comme nous allons le voir ci-après.

\section{L'ÉPISODE DE LA MARCHE SUR LES EAUX}

Cet épisode fait partie d'un ensemble de récits de conversion après la période de l'éveil. Il met en scène, à Uruvilvā, le Buddha qui, ayant fait halte non loin de l'ermitage de l'ascète à tresses Kāśyapa, entouré de ses cinq cents disciples, parvient à l'aide de ses pouvoirs surnaturels à dompter le serpent venimeux local, à accorder l'hospitalité aux quatre grands rois divins puis au roi des dieux Śakra-Indra ainsi qu'à Brahmā sahamppati, à faire un aller-retour éclair dans I'Uttarakuru, à laver des haillons dans un étang que Śakra créa expressément pour lui à cette occasion, à congédier Kāśyapa mais à arriver avant lui à sa
[14] Dans la traduction chinoise du Buddhacarita (佛所行讚, Fo suoxing zan) due à Dharmakșema, vers 420 ap. J.-C.), il est seulement spécifié que le Buddha procéda à la manifestation de ses nombreux pouvoirs surnaturels (神變, shénbiàn, T. 192, vol. 4, p. 31c8) ou ṛddhiprātihārya (pā[li], iddhipāțihāriya).

[15] PTS, Jātaka, vol. 2, Sīlānisaṃsajātaka 190, p. 111. [16] T. 211, vol. 4, p. 580a1 et suivantes.
[17] La quadruple représentation des pieds du Buddha et de deux oies sauvages sur un bas-relief du stūpa d'Amarāvatī a parfois été identifiée à l'épisode de la marche sur les eaux. Il s'agit plutôt ici de la marche sur la rive de la Nairañjanā avant l'éveil. Cf Buddhacarita 12.108. BARRETT 1954, p. 65-66 et planche XXIb ; KNOX 1992 , p. 50-51.

[18] FOUCHER 1987, p. 219.

[19] MaRSHALL 1918, p. 65. 


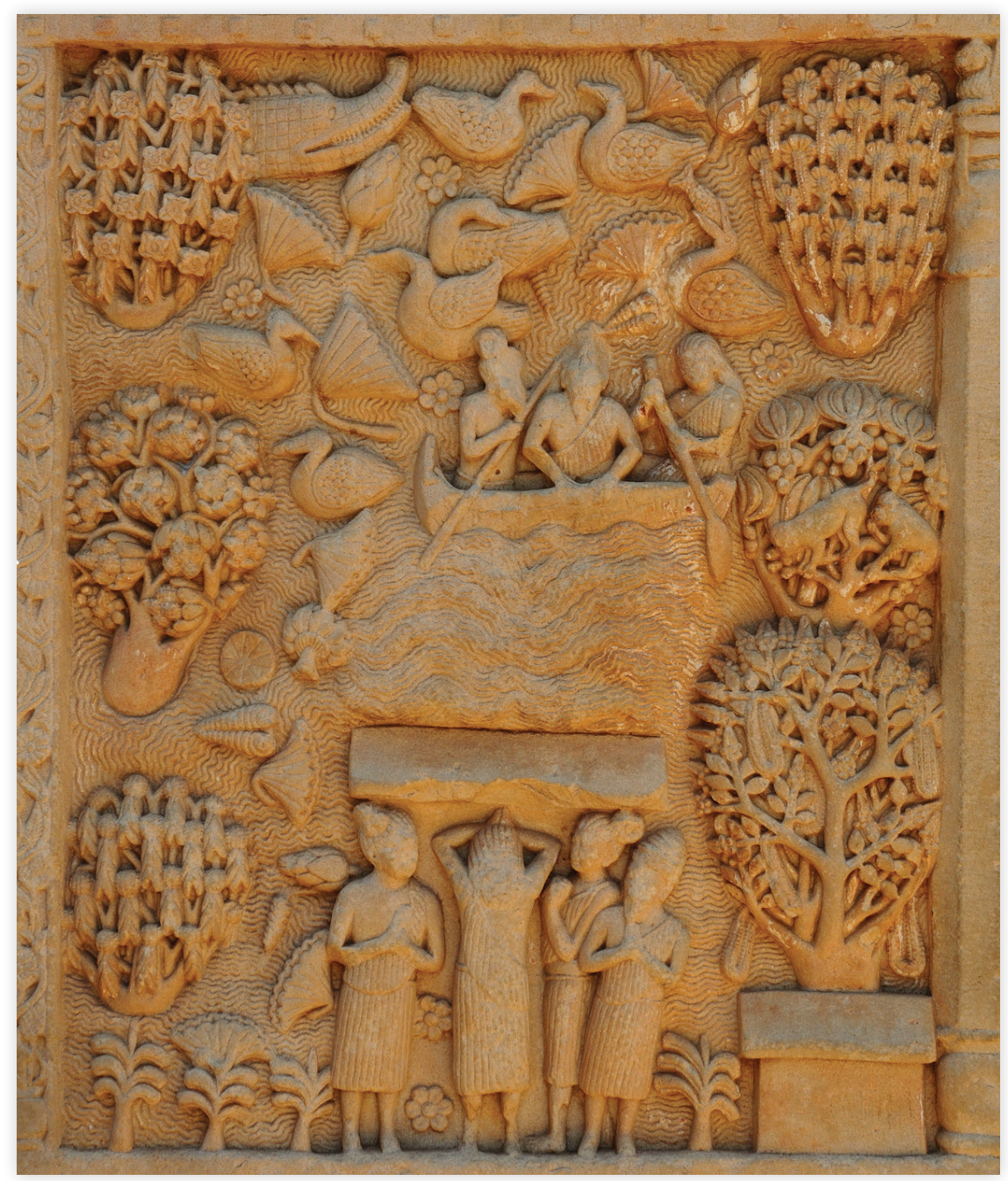

Figure 1:

Porte est du stūpa de Sāñcī. La marche sur les eaux du Buddha

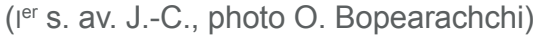
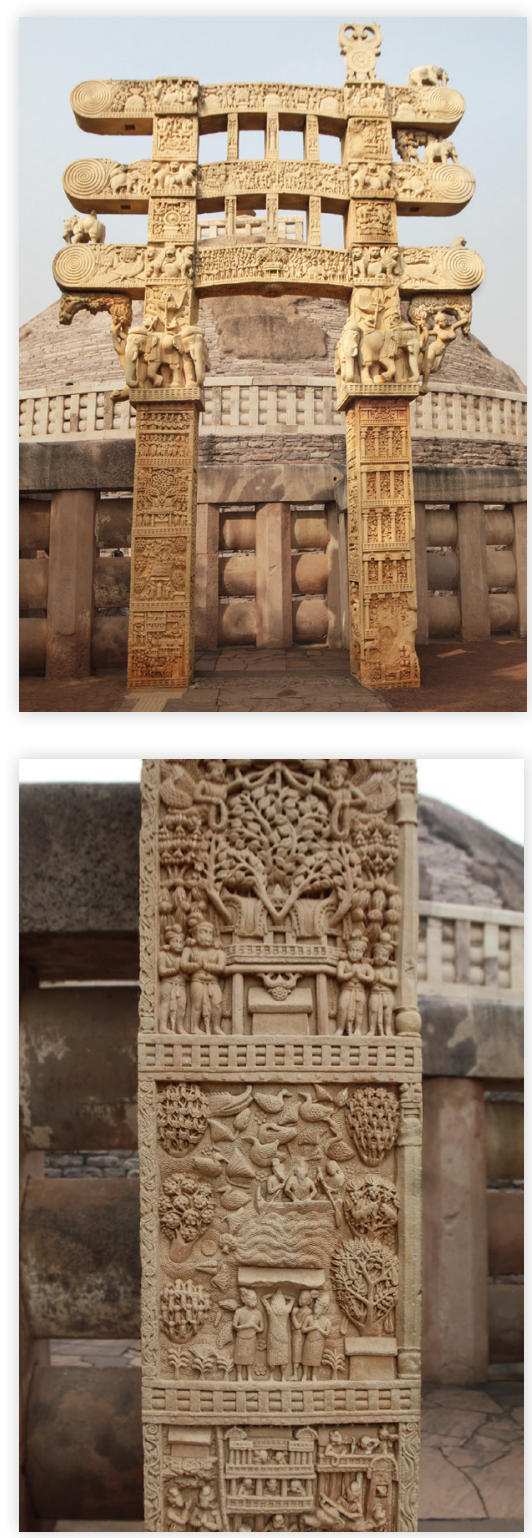

hutte, à empêcher ses disciples de couper du bois avec des haches et ainsi d'allumer un feu sacrificiel, à marcher sur les eaux lors d'une vaste inondation, puis à s'élever dans les airs avant de confondre le vieil ascète au sujet de sa prétention à se considérer lui-même parfaitement accompli (arhant) dans le domaine de l'ascétisme et de la délivrance du cycle des transmigrations. Tous ces prodiges s'imposèrent à Kāśyapa tout autant qu'à ses cinq cents disciples, et tous finirent par se convertir à la doctrine du Buddha.

Néanmoins, si les sources s'accordent entre elles, certains éléments narratifs diffèrent. C'est le cas du prodige qu'accomplit le Buddha pour survivre à une terrible inondation. Les textes rapportent tous qu'un grand nuage hors de saison (mahākālamegho [20]) ou noir [21] (黑雲大, hēi yún dà, T. 1421, p. 109a21； 大黒雲, dà hēi yún, T. 1428, p. 796a13 et T. 125, p. 621c2) déversa une pluie intense, pendant sept jours (七日, qī rì, T. 1421, p. 109a21), qui fit déborder de son lit la rivière Nairañjanā (連若大河, lianruo dàhé, T. 125 , p. 621c2-3 ; 尼連禪河, nilianchan hé, T. 1421, p. 109a23) entraînant alors une vaste inondation (mahāudakavāhako; 浩成一水, hào chéng yī shuǐ, T. 1421, p. 109a22; 潦水, liáo shuǐ, T. 1428， p. 796a14). Inquiet, Kāśyapa monta dans un bateau (nāvāya ; 船, chuán, T. 1421, p. 109a23) fait d'un tronc d'arbre (一樹船, yī shù chuán, T. 1428, p. 796a16), accompagné de nombreux disciples (sambahulehi jațilehi saddhiṃ), au nombre de cinq cents (五百弟子，wǔbǎi dìzí, T. 125, p. 621c5), ou bien se rendit sur les bords de la Nairañjanā (往至河所,

[20] PTS, Vinaya-pițaka, vol.1, Mahāvagga 1.20.16, p. 32.

[21] Les traducteurs chinois semblent avoir compris le composé mahākālamegho par mahā+kāla+megho ( « un grand nuage noir »), là où celui-ci indiquait qu'« un grand nuage hors de saison » (mahā+akāla+megho) s'abattit sur la région. Cet élément narratif avait pour finalité de souligner la soudaineté de l'événement en dehors de la période de la saison des pluies. 
wăngzhì hé suǒ, T. 125, p. 621c5), afin de s'assurer que le Buddha n'eût pas été emporté par le flot dévastateur. Il vit alors le Buddha qui soit marchait sur les eaux de la rivière Nairañjanā (尼連禪河水上經行, Nilianchan hé shuisshàng jīngxíng, T. 1421, p. 109a24) sans que ses pieds ne soient mouillés (爾時世尊在水上行 脚不爲 水所, ěrshí shìzūn zài shuǐshàng xíng jiǎo bù wéi shuǐ suǒ, T. 125, p. 621c5-6), soit, qui, après avoir repoussé les eaux (udakaṃ ussāretvā), se promenait sur une bande de terre sèche (majjhe reṇuhatāya bhūmiyā cańkamantam； 世尊爾時在外露地經行 地燥如 舊, shìzūn ěrshí zàiwài lòudì jīngxíng dì zào rú jiù, $T$. 1428, p. 796a16-17). À ce constat, Kāśyapa lui reconnut la capacité à user de grands pouvoirs surnaturels (mahiddhiko ; 神, shén, T. 1421, p. 109a24 et T. 125, p. 621c9; 神足, shénzú, T. 1428， p. 796a20) sans pour autant lui accorder la qualité d'arhant (arahā ; 阿羅 漢, āluóhàn, T. 1428, p. 796a20-21) ou d'avoir atteint la voie de l'arhant (阿羅漢道, āluóhàn dào, T. 1421, p. 109a26) ou encore la vérité de la voie (道眞, dào zhēn, T. 125, p. 621c10) à son égal. Après s'être élevé dans les airs (vehāsam abbhuggantvā; 飛昇虚空, fēi shēng xū kōng, T. 1421, p. 109a27) puis avoir surgi dans la barque (nāvāya paccutțhāsi), le Buddha pointa la prétention de l'ascète brāhmanique à se déclarer arhant lors même qu'il ne l'était point (neva kho tvam Kassapa arahā ; 汝非羅漢, rǔ fēi luóhàn, T. 1421, p. 109a27 [22]) et qu'il n'était aucunement capable de marcher sur l'eau comme il s'en vantait ouvertement (吾能在水上行, wú néng zài shuǐshàng xíng, T. 125, p. 621c14) une fois invité à y marcher ensemble (可 共在水上行耶, kě gòng zài shuǐshàng xíng yé, T. 125, p. 621c14-15). Kāśyapa reconnut publiquement son erreur et se convertit à la doctrine du Buddha. À son tour, la troupe de ses disciples en fit autant.

Pour mieux saisir la spécificité de cet exploit, il convient de prendre également en considération les histoires dans lesquelles des disciples du Buddha traversent une rivière en marchant sur l'eau. Ces épisodes appartiennent également à des récits de conversion. Dans la version chinoise glosant le Dharmapāda et traitant de la puissance de la foi (信, xìn ; skt śraddhā), le Buddha ne parvint pas à convertir à sa doctrine les habitants d'un hameau implanté sur la rive d'un large et profond fleuve s'écoulant au sud-est de la ville de Śrāvastī. À I'aide de ses pouvoirs supranormaux, il créa alors un

[22] Cf. 非阿羅漢, fēi āluóhàn, T. 1428, p. 796b4 汝亦非阿羅漢, rǔ yì fēi āluóhàn, T. 125, p. 621c10-11.

[23] PTS, Jātaka, vol. 2, Sīlānisaṃsajātaka 190, p. 111.

[24] sayyathāpi nāma udake pi abhidyamāno gacchat tadyathāpi nāma pṛthivīyaṃ, Mhv 3.410. homme (化作一人, huàzuò yīrén) qui s'avança vers eux de la rive sud en marchant sur l'eau (足行水上， zúxíng shuǐshàng). Étonnés de le voir ainsi franchir ce large et profond fleuve, les riverains apprirent de lui qu'il avait demandé aux pêcheurs de la rive sud s'il pouvait traverser sans barque, pressé qu'il était de rencontrer son sauveur. Ce à quoi les bateliers répondirent que le fleuve n'était guère profond. Il les crut et ce fut ainsi qu'il marcha sur l'eau dans l'objectif d'atteindre la rive d'en face pour entendre les paroles du Buddha. Ce dernier déclara alors que « la foi fait traverser les eaux profondes [de l'existence] 》(信能渡淵, xìn néng dù yuān, T. 211, p. 580a19) et les riverains se convertirent enfin à sa doctrine menant à l'autre rive, celle de la délivrance du cycle des transmigrations (sampāara).

L'auteur de l'introduction du Sīānisamsajātaka, qui a pour sujet la foi (saddhā) et la conduite morale (sīla), raconte, quant à lui, comment un dévot (upāsaka) arrivant le soir sur la rive de l'Aciravatī et ne voyant aucun passeur, prit comme point d'appui [de concentration] le Buddha (buddhārammaṇam). « Ses pieds ne s'enfoncèrent pas dans l'eau comme s'il eût marché sur la surface du sol » (pādā udakamhi na osīdiṃsu so pațhavītale gacchanto viya [23]). Mais, parvenu au milieu de la rivière, il remarqua les vagues et, sa concentration s'affaiblissant, ses pieds s'enfoncèrent dans l'eau. Il redoubla alors d'effort dans sa concentration sur les vertus du Buddha (buddhaguna) et parvint finalement sur l'autre rive afin d'y rencontrer le Buddha prêchant la doctrine.

Enfin, dans le Mahāvastu, à la demande du Buddha, Yaśoda (pā. Yasada), jeune converti qui devint le septième arhant, dévoila ses pouvoirs supranormaux à sa famille venue le chercher afin de la convertir à la doctrine du maître. Parmi ses nombreuses démonstrations édifiantes, Yaśoda, sans fendre les eaux sous son poids, marcha dessus comme s'il s'agissait de la terre ferme [24].

L'histoire rédactionnelle de l'épisode de l'inondation soudaine montre que les auteurs ne s'accordaient pas sur le moyen échappatoire grâce auquel le Buddha parvint à ne pas être emporté par le flot dévastateur. Les Theravādin avançaient qu'il avait reflué les eaux et put ainsi marcher sur une bande de terre sèche, les Dharmaguptaka qu'il avait simplement marché en terrain découvert, les Mahīśāsaka et l'auteur de I'Ekottarāgama qu'il avait marché sur l'eau. En 1963, dans ses Recherches sur la biographie du Buddha, André Bareau (1921-1993), qui privilégiait souvent une lecture rationalisante de ce type de récit, avait supposé une bouddhisation d'un ancien lieu de culte établi après un événement qui aurait été regardé par la population locale comme relevant d'un miracle : 
« L'origine de cet épisode peut avoir été un événement qui avait frappé l'esprit des paysans d'Uruvilvā et leur avait fait crier au prodige : lors d'une inondation consécutive à des pluies particulièrement violentes, un homme aurait été épargné en se réfugiant sur une portion de terrain légèrement surélevée et qui, par chance, n'était pas recouverte par les eaux. On a dû alors attribuer ce prodige au pouvoir surnaturel de cet homme, surtout si celui-ci était un ermite religieux, puis, le temps passant, on a pu confondre ce personnage anonyme avec ce Kāśyapa thaumaturge autour duquel se cristallisaient peu à peu toutes les légendes locales, et, plus tard encore, au temps des pèlerinages bouddhiques, on a attribué ce miracle au Bienheureux lui-même.

On peut penser en outre que I'on ne manquait pas de montrer aux pieux visiteurs, avides de retrouver partout des souvenirs précis, visibles et tangibles, de la vie du Buddha, le léger tertre qui passait pour avoir servi de théâtre à ce prodige. Une fois de plus, il semble qu'un détail topographique des environs immédiats d'Uruvilvā ait été à I'origine d'un épisode de la légende du Buddha » [25].

À partir d'une telle lecture rationalisante, il en déduisit que la version des Dharmaguptaka apparaissait comme la plus naturelle. À leur suite, les Theravādin auraient fait intervenir la puissance surnaturelle du Buddha afin de faire refluer les eaux, puis les Mahīśāsaka n'auraient nullement hésité, quant à eux, à le faire directement marcher sur l'eau. Toujours dans un souci de rationalité, il en conclut que «sans doute pourrait-on expliquer cette dernière version en disant que le héros se promenant sur un haut fond à peine recouvert par l'eau, semble marcher à la surface de celle-ci, mais cette justification est rendue inutile par les deux autres récits. Ce sont donc bien les Mahīśāsaka qui, cette fois, exagèrent l'ampleur du miracle attribué au Buddha et qui paraissent nous donner la version la plus récente des trois » [26].

Cette tentative d'exégèse du canon scripturaire bouddhique ne peut emporter la conviction dès lors que des facteurs historiques et symboliques sont assurément à l'origine de la construction du dit épisode. Car, en partant d'une analyse textuelle qui considère au fondement du récit un événement naturel, $A$. Bareau en est finalement arrivé à privilégier la version des
Dharmaguptaka et à voir dans celle des Mahīśāsaka l'aboutissement de son histoire rédactionnelle par des ajouts d'éléments narratifs tenant du merveilleux. Or, la chronologie des variantes doit être regardée sous un angle d'approche différent.

Il nous semble préférable de garder à l'esprit, comme le soulignait A. Foucher, que les représentations figurées ont parfois été à l'origine de variantes en fonction de leur compréhension et de leur réception dans les milieux bouddhiques eux-mêmes. Le bas-relief de la porte est du stūpa de Sāñcī offre une telle hypothèse d'explication. En Inde centrale, au I ${ }^{\mathrm{er}} \mathrm{s}$. av. J.-C., le Buddha ne fit l'objet d'aucune représentation anthropomorphe, mais au contraire d'un ensemble de figurations symboliques. Ainsi, lorsque les artistes en vinrent à représenter le Buddha marchant sur les eaux de la rivière Nairañjanā, ils ne purent sculpter symboliquement que sa seule promenade ( $p a \overline{\text {. }}$. cańkama, skt cańkramaña) méditative à la surface de l'eau qu'ils rendirent en laissant une bande de pierre, vierge de toute ligne ondulée. C'est également le cas des bas-reliefs des portes est et nord du stūpa de Sāñcī sur lesquels est représentée, par cette même bande de pierre lisse, la présence du Buddha à Kapilavastu respectivement lors de la procession du roi Śuddhodana [27] et lorsqu'il accomplit I'exploit de marcher dans les airs [28]. La marche sur l'eau était d'autant plus aisée à figurer de cette façon que sa formulation verbale était couplée à la comparaison (upamā) récurrente « comme sur la terre ferme ».

Ainsi, le récit des Dharmaguptaka ne donne certainement pas « la version la plus naturelle de l'événement », selon A. Bareau, mais une mauvaise interprétation de la représentation symbolique artistique de la marche sur les eaux telle qu'elle est encore aujourd'hui attestée sur le bas-relief du stūpa de Sāñcī. Il en est de même des Theravādin qui, pour rendre plus logique la présence de cette bande de pierre non inondée au milieu des flots, ajoutèrent que le Buddha avait au préalable fait refluer les eaux. Ceci induit que la version des Mahīśāsaka, loin de toute exagération, et celle de l'Ekottarāgama conservent toutes deux I'élément narratif premier, à savoir que le Buddha marcha sur l'eau. Cet exploit attribué au Buddha est confirmé dans la tradition bouddhique par les récits des disciples marchant à leur tour sur les eaux et par les Vinaya réglant la question des pouvoirs surnaturels. Il convient dès lors d'en saisir et la portée symbolique et les obligations disciplinaires. 


\section{DU POUVOIR SURNATUREL DES ŚRAMAṆA À LA CONDITION DE L'ARHANT}

En premier lieu, la marche sur les eaux est à replacer dans son contexte large, celui de la manifestation des pouvoirs supranormaux du Buddha que lui a procuré son éveil (bodhi) et qui participent à l'édification de son entourage pour amener ce dernier à se convertir à sa doctrine. L'auteur ou les auteurs des récits de conversion successifs des trois frères Kāśyapa ont donc utilisé dans l'épisode qui nous intéresse la manifestation publique de l'un d'entre eux, à savoir la marche sur les eaux, couplé à la symbolique du flot (ogha) dévastateur du saṃsāra et de sa traversée salvifique.

En Inde ancienne, si les brāhmanes revendiquaient le pouvoir de faire fléchir les dieux (deva) par leur savoir sacré (veda) et leurs rites sacrificiels (yajña), les ascètes (śramaṇa) étaient dits posséder des pouvoirs surnaturels ou réalisations (rddhi) obtenus après de longues années d'échauffement ascétique ou mortification ardente (tapas). La littérature de langues indo-ārya est emplie de ces renonçants, appelés siddha ou accomplis, aux pouvoirs magiques (siddhi) si puissants que même les deva sont dits s'en méfier. Certains traités d'ascétisme, tels les Yogasūtra de Patañjali (II ${ }^{\mathrm{e}}-\mathrm{III}^{\mathrm{e}}$ s. apr. J.-C. ?), ou bien quelques Purāna fournissent des listes de ces siddhi, vibhūti, rddhi, perfections, réalisations, manifestations de puissance qu'entraîne, selon leurs auteurs, la pratique des observances et des restrictions ascétiques. Les bhikșu bouddhistes ont dressé leur propre liste plus ou moins canonique. Le Sāmaññaphalasutta du Suttapițaka de l'école du Theravāda énumère ces pouvoirs surnaturels comme suit :

«Quand sa pensée est ainsi absorbée, toute pure, toute nettoyée, sans tache, exempte d'impuretés, devenue souple, prête à agir, stable, arrivée à l'impassibilité, il donne une impulsion à sa pensée et l'oriente vers les pouvoirs merveilleux. Il éprouve en lui tel ou tel des pouvoirs merveilleux : étant un il

[29] PTS, Dhīga Nikāya, vol. 1, sutta 2, p. 78. Comme nous I'avons vu en Mahāvastu 3.410, I'histoire du jeune Yaśoda fut l'occasion pour les auteurs Mahāsāmnghika d'énumérer également la liste canonique des pouvoirs surnaturels (anekavidhāṃ ṛddhiṃ, Mhv 3.409) qu'il acquit auprès du Buddha.

[30] Canon bouddhique pāli 1989, p. 69.

[31] PTS, Dīgha Nikāya, vol. 1, sutta 2, p. 75.

[32] Vision de la vraie connaissance, production d'un corps spirituel, acquisition de pouvoirs surnaturels, pra- devient multiple ; étant multiplié il devient un ; il devient visible, invisible, franchit les murs, franchit les remparts, franchit les montagnes sans en être gêné, comme si c'était de l'air libre ; il s'immerge dans la terre et en émerge comme dans de I'eau ; il va sur l'eau sans qu'elle se brise, comme sur la terre (udake pi abhijjamāno gacchati seyyathā pi pațhaviyam [29]) ; il marche dans les airs avec les jambes croisées comme un oiseau avec ses ailes ; il prend en mains, il touche la lune et le soleil, ces deux êtres merveilleux, prestigieux, et avec son corps il étend sa volonté jusqu'au monde de Brahman » [30].

Marcher sur l'eau comme sur la terre ferme fait donc partie d'une liste de pouvoirs surnaturels à l'égal de sa contrepartie : plonger dans la terre comme s'il s'agissait de l'eau. Ces pouvoirs surnaturels sont obtenus par le bhikșu lorsqu'il a atteint le quatrième et dernier stade de la concentration (catutthajjhānaṃ [31]) et sont donc « le fruit visible de son état de religieux » (sandițțikaṃ sāmaññaphalaṃ). Néanmoins, la longue énumération croissante des fruits [32] s'achève, selon l'auteur du Sutta, par les quatre nobles vérités fondatrices de la doctrine bouddhique. Ainsi, I'ultime et véritable fruit de la vie d'un religieux-mendiant bouddhiste n'est nullement I'acquisition de pouvoirs surnaturels, mais bien plutôt la reconnaissance de l'origine de la souffrance et de la voie menant à sa destruction et ouvrant à I'extinction finale ou nirvāna sans plus de retour transmigratoire : «Quand il sait cela, quand il voit cela, son esprit est libéré des nocivités du désir, son esprit est libéré des nocivités de l'être, son esprit est libéré des nocivités du non-savoir. Quand il est libéré, il comprend qu'il y a libération. Il reconnaît que la renaissance est détruite, que la vie brahmique est vécue, que la tâche est achevée, qu'il n'y a pas de retour ici-bas. [...] Il n'y a point, grand roi [33], d'autre fruit visible de l'état de religieux [qui soit] plus haut et plus sublime que ce fruit visible de l'état de religieux $\gg[34]$. tique du son céleste, pénétration dans la pensée d'autrui, mémoire de ses propres existences passées, connaissance de la vie d'autrui.

[33] Il s'agit d'Ajātasattu Vedehīputta, roi du Magadha, qui tua son père Bimbisāra, ami du Buddha, et qui finit, selon la tradition bouddhique, par se convertir à la doctrine après avoir entendu de la bouche du Buddha les fruits rendus visibles de l'état de religieux bouddhistes.

[34] Canon bouddhique pāli 1989, p. 74-75. Dans les Yogasūtra, les siddhi sont considérés également comme des obstacles (upasarga) au samādhi. 
En second lieu, nous avions vu que cet épisode de la vie du Buddha provenait des Vinaya des écoles anciennes. Or, les règles disciplinaires sont très strictes au sujet du faire valoir de tels pouvoirs surnaturels. Aussi, un bhikșu qui prétendrait posséder de tels pouvoirs surhumains (uttarimanussadhammam [35]) afin d'obtenir notamment des dons de quelque nature que ce soit, s'exposerait à l'exclusion définitive de la communauté bouddhique (asaṃvāso), vaincu (pārājiko) qu'il serait alors par ses propres désirs. Une telle prétention est d'ailleurs mise à pied d'égalité avec les trois précédents pārājika relevant des relations sexuelles, du vol et du meurtre. C'est dire combien devaient être préoccupants pour le saṃgha les abus de confiance occasionnés par certains bhikșu vis-à-vis d'autrui et surtout des dévots et dévotes qui subvenaient à tous leurs besoins d'existence quotidiens.

Si le récit de conversion de Kāśyapa d'Uruvilvā repose donc sur la mise en scène des pouvoirs supranormaux attribués à l'Éveillé, il n'en demeure pas moins que sa portée disciplinaire est assurément première. En effet, tous les prodiges réalisés par le Buddha ne suffirent aucunement à impressionner le vieil ascète. Bien que ce dernier reconnût la puissance surnaturelle du Buddha, jamais il ne lui accorda le fait d'avoir atteint l'état d'arhant à son égal. Or, c'est bien dans cette reconnaissance du statut d'accompli ou de méritant (arhant) que repose la double portée de ce récit de conversion et le prodige de la marche sur l'eau. Non seulement les prodiges accomplis ne sont pas garants de l'état d'arhant, mais encore Kāśyapa commit la faute de prétendre être un arhant auprès de ses cinq cents disciples lors même qu'il n'en était pas un aux yeux du Buddha :

neva kho tvaṃ
Kassapa arahā, na
pi arahattamag-
gam samāpanno,
sā pi te pațipadāa
natthi, yāya tvaṃ
arahā vāa assa
arahattamaggaṃ
vā samāpanno
'ti [36]

Ainsi apparaissent les finalités d'une telle construction de la figure du Buddha dans des compositions relevant des règles disciplinaires. Le face-à-face antithétique visant à montrer la supériorité du Buddha sur Kāśyapa d'Uruvilvā, archétype de l'ascète à tresses brāhmanique, fut l'occasion pour les auteurs bouddhistes d'énumérer les pouvoirs supranormaux qu'engendre le quatrième et dernier stade de la concentration, grâce auquel Śākyamuni accéda à I'éveil, comme libération du monde phénoménal et des contraintes que ce dernier impose à l'être humain dans le domaine de la matière (mahābhūta), tout en réaffirmant que le plus haut fruit de la vie de religieux demeure la parfaite connaissance des quatre nobles vérités qui conduisent à la condition de l'arhant et donc à l'extinction finale de toute souffrance.

Dans le contexte bouddhique, la marche sur les eaux effectuée par le Buddha, au-delà du prodige comme moyen de conversion, relève donc de l'idée plus générale que l'arhant est, de son vivant, pleinement affranchi des quatre éléments (terre/prthivī, eau/ āpas, feu/tejas, air/vāyu) qui composent le monde des désirs et qu'il en échappera définitivement à sa mort. Et, si le Buddha, monté sur la barque de sa doctrine (dharmanāvam), a su traverser le flot des transmigrations (saṃsāraoghe), a su franchir les vagues des existences (bhavārṇavaṃ), si lui, ce batelier (nāvika), a invité autrui à le rejoindre sur l'autre rive, celle de l'extinction, de "I'heureuse terre ferme où il n'y a plus ni vieillesse ni mort » (sthale ajarāmane śive [38]), pour être sauvé et se sauver [39] des renaissances incessantes, il ne fait aucun doute que, plus encore, certains rédacteurs bouddhistes, maîtres dans l'art de la métaphore (uprekșā, rūpaka), en sont également venus à faire traverser les disciples qui, en prenant appui sur les seules vertus (guna) du Buddha et forts de leur foi (śraddhā) en l'efficience de sa doctrine, réitérèrent son exploit de marcher sur les eaux des existences infinies.

[35] PTS, Vinaya Pițaka, vol. 3, Suttavibhaniga, pārājika 4, p. 90. Cf. « uttari manudharme » en Bhikșuṇivinaya 183.7.

36] PTS, Vinaya-pițaka, vol.1, Mahāvagga 1.20.17, p. 32.

37] Dans la doctrine bouddhique l'arhant est le titre donné au bhikșu qui est parvenu au plus haut stade de la carrière des auditeurs (srāvaka), c'est-à-dire qui a vaincu les dix liens (samyojana) à rétributions basse et haute dus aux passions et qui a atteint de son vivant l'extinction (nirvāna). A sa mort, il parvient à l'extinction totale et définitive (parinirvāna) sans plus de résidus karmiques et est donc assuré de ne plus être sujet à aucune renaissance dans le monde des désirs (kāmadhātu).

[38] Lalitavistara 15.55-57g. Voir Duc氏euR 2017, p. 190.

[39] Depuis la période du Rgveda au moins, la notion même de salut repose sur l'idée de traverser (pr- ou tr-) d'une rive à l'autre, d'atteindre sain et sauf l'autre rive. Lors des rites védiques solennels, par exemple, le sacrifiant (yajamāna), une fois monté sur le bateau à rames qu'est le sacrifice dirigé par les officiants (rtvij), traverse de la rive du monde des hommes à la rive de l'océan céleste des entités diurnes (deva). Voir DUCEUR 2008. 


\section{BIBLIOGRAPHIE}

BAREAU, André, 1963, Recherches sur la biographie du Buddha dans les Sūtrapițaka et les Vinayapițaka anciens : De la quête de l'éveil à la conversion de Śāriputra et de Maudgalyāyana, École française d'Extrême-Orient, Paris.

BARRETT, Douglas, 1954, Sculptures from Amarāvatī in the British Museum, British Museum, London.

BRown, William Norman, 1928, The Indian and Christian Miracles of Walking on the Water, Chicago, Open Court.

Canon bouddhique pāli, 1989, texte et traduction Suttapițaka Dīghanikāya par Jules BLoch, Jean FiLLIOZAT, Louis Renou, Tome I, fascicule I, Paris, J. Maisonneuve.

Carus, Paul, 1897, Buddhism and Its Christian Critics, Chicago, Open Court.

DucceUR, Guillaume, 2008, « Passing through Flood Waters in Vedic Thought », The Journal of Indo-European Studies, vol. 36/1-2, p. 67-78.

DucGUR, Guillaume, 2011, « Le Buddha historique et canonique », in DuccUR, Guillaume, 2011, Initiation au bouddhisme, Ellipses, Paris, p. 263 à 373.

Duccur, Guillaume, 2014, «Le bodhisattva à la salle d'écriture : histoire rédactionnelle et datation », in Revue de I'Histoire des religions, tome 231/3, p. 385-424.

Duccur, Guillaume, 2017, « Du mont Potala au mythe du déluge dans l'Asia Polyglotta de J. Klaproth », dans Christophe Vielle, Christian CANNuYer et Dylan EsLeR (éd.), Dieux, génies, anges et démons dans les cultures orientales et florilegium indiae orientalis Jean-Marie Verpoorten in honorem, coll. « Acta Orientalia Belgica », vol. 30, p. 185-195.

Duccur, Guillaume, 2019, «Śākyamuni émacié ou la critique de la pratique des austérités », in Revue de I'histoire des religions, tome 236/1, p. 11-40.

Foucher, Alfred, 1949, La vie du Bouddha d'après les textes et les monuments de l'Inde, Payot, Paris.

Foucher, Alfred, 1987, La vie du Bouddha d'après les textes et les monuments de I'Inde, J. Maisonneuve, Paris (1 ère éd. Payot 1949), p. 219.

GARBE, Richard VoN, 1914, Indien und das Christentum. Eine Untersuchung der religionsgeschichtlichen Zusammenhänge, Tübingen, J. C. B. Mohr.

KNox, Robert, 1992, Amarāvatī. Buddhist Sculpture from the Great Stûpa, British Museum Press, London.

LILLIE, Arthur, 1893, Influence of Buddhism on Primitive Christianity, Ch. Scribner's Sons, New-York.

MARSHALL, John, 1918, A Guide to Sanchi, Superintendent Government Printing, Calcutta.

Oldenberg, Hermann, 1903, Le Bouddha, sa vie, sa doctrine, sa communauté, traduit de l'allemand par A. Foucher, $2^{\mathrm{e}}$ édition, F. Alcan, Paris.

Palumbo, Antonello, 2013, An Early Chinese Commentary on the Ekottarika-āgama, Dharma Drum Publishing Corporation, Taipei.

SaIntyves, Pierre, 1922, Essais de folklore biblique. Magie, mythes et miracles dans l'Ancien et le Nouveau Testament, Paris, É. Nourry, p. 337-350.

SENART, Émile, 1875, Essai sur la légende du Buddha, son caractère et ses origines, extrait du Journal asiatique (années 1873-1875), Imprimerie nationale, Paris ( $2^{\mathrm{e}}$ éd. revue et suivie d'un index, E. Leroux, Paris, 1882).

TOURNIER, Vincent, 2017, La formation du Mahāvastu et la mise en place des conceptions relatives à la carrière du bodhisattva, École française d'Extrême-Orient (« Monographies, n 195), Paris. 